\title{
Write-Read 3D Patterning with a Dual-Channel Nanopipette
}

\section{Supporting Information}

\author{
Dmitry Momotenko,,$^{1, *}$ Ashley Page, ${ }^{1,2}$ Maria Adobes-Vidal ${ }^{1}$ and Patrick R. Unwin ${ }^{1, *}$. \\ ${ }^{1}$ Department of Chemistry and ${ }^{2}$ MOAC Doctoral Training Centre, University of Warwick, \\ Coventry, CV4 7AL, United Kingdom
}

*p.r.unwin@warwick.ac.uk

*d.momotenko@warwick.ac.uk 


\section{SI-1. Estimation of cross-contamination in the nanopipette barrels}

Filling the double-barrel glass nanopipettes with electrolyte solutions of different composition may result in a cross-contamination of the solutions in the barrels from the liquid droplet formed at the probe tip. To estimate the degree of such contamination, a simple mathematical treatment can be developed. First of all, one can consider spherical diffusion of the species from the probe tip into the pipette. For simplicity, in the first approximation one can provide an estimate for the diffusion into a single-barreled pipette, as schematically illustrated in Figure S-1.
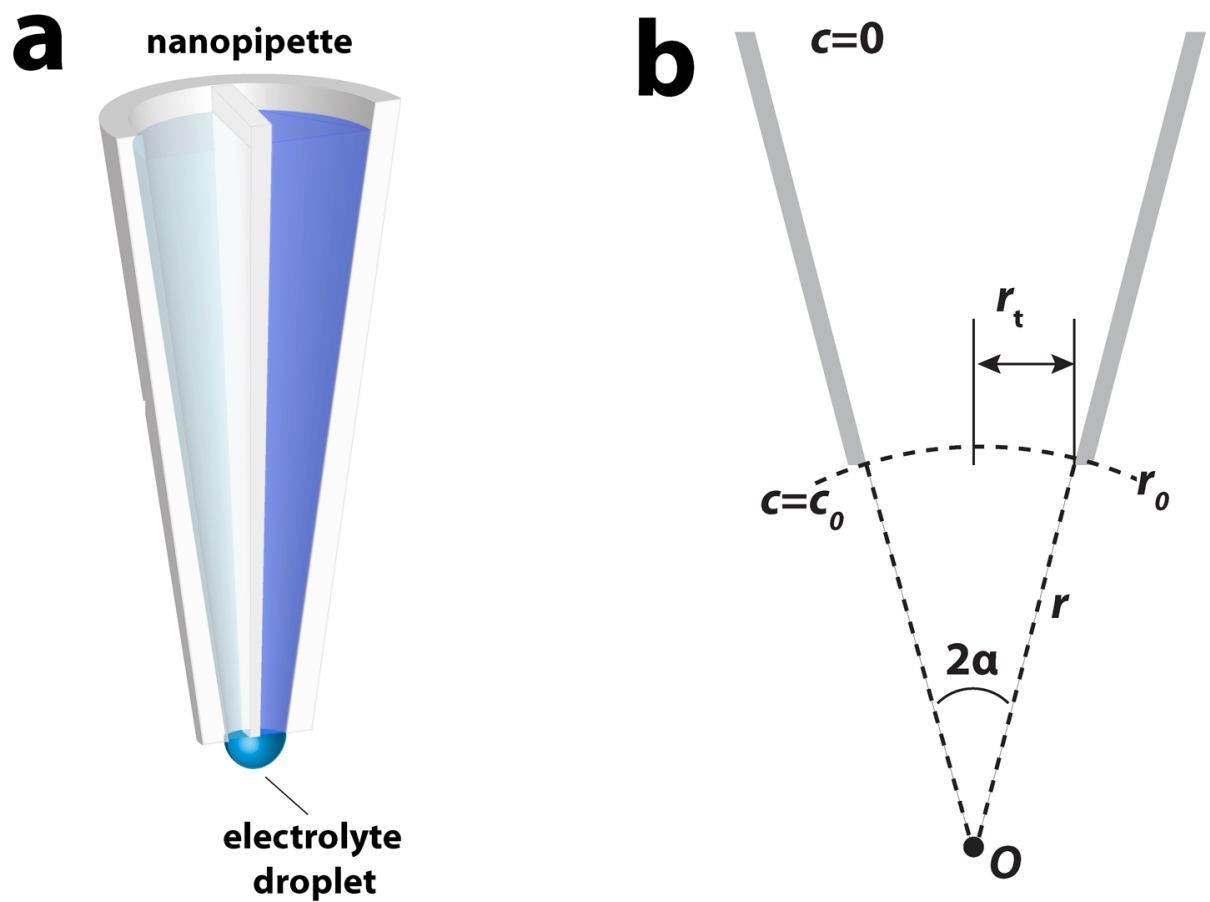

Figure S1. a) Schematic representation of the double-barrel nanopipette with an electrolyte droplet at the tip and (b) the geometrical interpretation of the mass transport in the nanopipette with concentration boundary conditions for modeling.

The model reasonably assumes the flux conservation equation applies (only masstransport, no chemical reactions involved):

$$
\nabla J=0
$$

where $J$ is the flux of species, given by the Fick's law:

$$
J=-D \nabla c
$$

$D$ and $c$ denote the diffusion coefficient and species concentration, respectively.

In spherical coordinates eq. 1 therefore reads 


$$
-D\left(\frac{\partial^{2} c}{\partial r^{2}}+\frac{2}{r} \frac{\partial c}{\partial r}\right)=0
$$

The solution of eq. 3 is

$$
c=-\frac{A}{r}+B
$$

where integration constants can be calculated from the boundary conditions $\left(A=c_{0} r_{0}\right.$ and $B=0$ as $c\left(r=r_{0}\right)=c_{0}$ and $c(r=\infty)=0 ; c_{0}$ denotes bulk concentration in the droplet). Therefore, the complete solution of eq. 3 reads

$$
c=\frac{c_{0} r_{0}}{r}
$$

The flux of species at $r=r_{0}$, obtained by differentiation of eq. 5

$$
J=\frac{D c_{0}}{r_{0}}
$$

allows the calculation of the amount of species entering the nanopipette within a time, $t$, taking into account the area $S$ of a spherical cap at $r=r_{0}$. The concentration change caused by the crosscontamination (the pipette volume, $V$, can be estimated knowing the capillary dimensions, such as internal diameter $d=0.9 \mathrm{~mm}$ and the length $l=5 \mathrm{~cm}$ )

$$
\Delta c=\frac{J S}{V} t=\frac{D c_{0}}{r_{0}} \frac{\pi\left(r_{t}^{2}+\left(r_{0}-r_{0} \cos a\right)^{2}\right)}{\pi\left(\frac{d}{2}\right)^{2} l} t
$$

where $a$ is the pipette semi-angle ( $8^{\circ}$ herein) and $r_{0}$ is given as

$$
r_{0}=\frac{r_{t}}{\sin a}
$$

One can assume the time required to fill both pipette barrels to be about $300 \mathrm{~s}$ (typically, this value is even lower in real experimental practice) and the concentration of species (with $D=10^{-9}$ $\mathrm{m}^{2} \mathrm{~s}^{-1}$ ) in the liquid droplet at the tip of $1 \mathrm{M}$ (which is, indeed, set very high, in order to calculate the upper limit of the cross-contamination effect). Under such assumptions, the concentration 
change due to the cross-contamination in the pipette reaches $82.9 \mathrm{nM}$. This is typically a negligible change for the 10 s to 100 s millimolar concentrations of electrolyte present in the nanopipette barrels. 